Para enlazar con este artículo / To link to this article:

http://dx.doi.org/10.6035/MonTI.2019.ne4.10

Para citar este artículo / To cite this article:

Martínez Tejerina, Anjana \& Samantha Sánchez Martínez. (2019) "El doblaje de acentos extranjeros y regionales en Los Simpson." En: Pérez L. de Heredia, María \& Irene Higes Andino (eds.) 2019. Multilingüismo y representación de las identidades en textos audiovisuales / Multilingualism and representation of identities in audiovisual texts. MonTI Special Issue 4, pp. 281-307.

\title{
EL DOBLAJE DE ACENTOS EXTRANJEROS Y REGIONALES EN LOS SIMPSON ${ }^{1}$
}

\author{
Anjana Martínez Tejerina \\ anjana.martinez@upf.edu \\ Universitat Pompeu Fabra \\ Samantha Sánchez Martínez \\ samantha.sanchez01@estudiant.upf.edu \\ Universitat Pompeu Fabra
}

\section{Resumen}

El doblaje de los acentos es un fenómeno frecuente en nuestras pantallas que no ha recibido, sin embargo, suficiente atención académica. El presente estudio compara la versión original con la versión doblada de la serie de animación The Simpsons (Matt Groening 1989- ) y analiza 32 personajes cuyo acento diverge del estándar. El análisis cuenta además con la experiencia en primera persona de María José Aguirre de Cárcer, la actual traductora de la serie al castellano. La investigación revela que factores como los obstáculos lingüísticos, las restricciones visuales o los estereotipos determinan la solución empleada. A pesar de que el uso de acentos está íntimamente relacionado con la identidad, los resultados obtenidos indican que la mayoría de las técnicas suponen, en mayor o menor grado, un cambio en la caracterización del personaje.

\begin{abstract}
"The dubbing of foreign and regional accents in The Simpsons"

Although the dubbing of accents is a common phenomenon on our screens, it has not received enough scholarly attention. This study compares the original version with the dubbed version of the animated series The Simpsons (Matt Groening 1989- ), and
\end{abstract}

1. El presente artículo ha sido realizado en el marco del grupo de investigación consolidado CEDIT (2014 SGR 843) del Departament de Traducció i Ciències del Llenguatge de la Universitat Pompeu Fabra. 
analyses 32 characters with a non-standard accent. The research is also based on the first-person experience of María José Aguirre de Cárcer, the current translator of the series to European Spanish. This investigation discloses that factors such as linguistic obstacles, visual restrictions or stereotypes determine the final solution. Despite the close link between accents and identity, the results obtained suggest that most translation techniques imply changes in characterisation to a greater or lesser extent.

Palabras clave: Acentos. Doblaje. Estereotipos. Multilingüismo. Traducción Audiovisual. Keywords: Accents. Dubbing. Stereotypes. Multilingualism. Audiovisual Translation. 


\section{Introducción}

"¿Mi voz ha sonado malévola? Pasa mucho con el acento ruso." Slava, capítulo 20 de la temporada 24 de la serie The Simpsons

Según la Real Academia Española, el multilingüismo consiste en la coexistencia de varias lenguas en un país o territorio. Una de las consecuencias de esta convivencia son los acentos. El hecho de que una persona tenga una lengua nativa distinta a la que está hablando genera un acento. Lippi-Green (2012: 46) explica esta situación en el caso del inglés de Estados Unidos:

When a native speaker of a language other than English learns English, accent is used to refer to the breakthrough of native language phonology into the target language. Thus we might say that an individual has a Welsh accent, or a Tagalog accent, because the phonologies of those languages influence the learner's pronunciation of U.S. English, and any effort to block the L2 accent will be accomplished with differing degrees of success.

Este acento originado por la lengua nativa vendrá a menudo acompañado, tal y como veremos en el corpus, de otras manifestaciones típicas del multilingüismo como el code-switching.

Aunque los acentos pueden reflejar características como el grupo social, en este estudio nos centraremos exclusivamente en las variantes diatópicas, que pueden explicitar lugares de procedencia más o menos extensos, es decir, continentes, países o regiones.

Con respecto al estado de la cuestión, la traducción audiovisual (TAV) del multilingüismo, un fenómeno cada vez más presente en nuestras pantallas, ha sido ampliamente analizada en los últimos años por autores como Corrius y Zabalbeascoa (2011), Heiss (2004) o de Higes-Andino et al. (2013). Sin embargo, no parece haber suscitado el mismo interés una de sus consecuencias más habituales: el acento. Quizá se debe a que esta manifestación está relacionada con aspectos fonológicos y no son resultado exclusivo de las decisiones del traductor, sino sobre todo de la interpretación de los actores. En cualquier caso, coincidimos con Steffensen (2012: 511) en que este objeto de estudio sí tiene cabida en la investigación sobre traducción audiovisual: "The dramaturgical use of accents can be considered an aspect of audiovisual translation 
(AVT) in the broad sense of the transfer of meanings between cultures through language".

Por otro lado, el uso de acentos en los medios audiovisuales está íntimamente relacionado con la representación de clichés tanto en la versión original (VO) como en la versión doblada (VD). Según autoras como Kozloff (2000: 26), la creación o perpetuación de estereotipos por medio del diálogo también merece una mayor atención:

Often overlooked is how much the speech patterns of the stereotyped character contribute to the viewer's conception of his or her worth; the ways in which dialect, mispronunciation, and inarticulateness have been used to ridicule and stigmatize characters have often been neglected.

Ante esta situación, surgen diversas preguntas. ¿Recurre el traductor a estrategias similares al traducir acentos que al traducir otro tipo de manifestaciones relacionadas con el multilingüismo? ¿Qué motivaciones y qué consecuencias tiene la elección de las distintas técnicas? ¿Cómo actúa ante la presencia de estereotipos en la VO? ¿Perpetúa la traducción los estereotipos? ¿Los subvierte? ¿Los modifica?

\section{El multilingüismo en los productos audiovisuales: representación, funciones y peligros}

El multilingüismo puede aparecer representado en el texto audiovisual de diversas maneras y en diversos grados. Mareš (2000a; 2000b; 2003) [cit. en Bleichenbacher (2008: 23-25)] distingue en los textos de ficción cuatro estrategias fundamentales: eliminación, señalización, evocación y presencia.

La eliminación, que es la estrategia más alejada de la realidad, consiste en sustituir el idioma que hablaría un personaje en la realidad y que no coincide con el idioma principal de la película por una variedad estándar de la lengua principal. Como no aparece ninguna pista lingüística, el espectador solo podrá reconocer el origen del personaje si es capaz de procesar indicios extralingüísticos. A este respecto, cabe recordar la capacidad semiótica de la imagen, tal y como destaca Whitman-Linsen (1992: 33):

We might go so far as to claim that the relatively versed viewer can recognize a number of nationalities watching solely the people on the screen with the sound eliminated. The Frenchman's shoulder movements and lower-lip idiosyncrasies, the American bearing of the body and free-swinging gait, and the Italian's 'talkative' hand signals can virtually not be mistaken for each other. 
La señalización consiste en que un personaje mencione de forma explícita su lengua $u$ origen en un comentario metalingüístico.

En la evocación el personaje habla la lengua principal del producto audiovisual, pero incluye múltiples interferencias de su lengua nativa. Bleichenbacher (2008: 60) afirma que, aunque estas interferencias pueden aparecer en cualquier plano lingüístico, se desarrollan con frecuencia en el plano fonológico, mediante acentos, y el léxico, mediante breves cambios de código o codeswitches, es decir, el hablante introduce palabras de su Ll. Esta estrategia sirve para indicar la procedencia del parlante, pues los acentos y las interferencias desvelan la Ll del personaje.

Por último, la presencia consiste en incluir una lengua distinta de la lengua principal de la película. Esta estrategia sería la más cercana a la realidad, pues supondría que, por ejemplo, cuando dos personajes rusos estén solos, hablen ruso y no inglés o castellano.

La variación lingüística, trasvasada por medio de estas cuatro estrategias, está presente a menudo en los productos audiovisuales, pues permite a guionistas y directores alcanzar determinados objetivos. Bleichenbacher (2008: 26) afirma que es imposible realizar un listado exhaustivo de las funciones que puede desempeñar el multilingüismo en un producto audiovisual, pero destaca tres categorías fundamentales: el realismo, el humor y la crítica social. En el presente apartado nos centramos en las dos primeras por su relevancia en el corpus objeto de análisis.

En primer lugar, cabría destacar la intención de reflejar una realidad de diversidad lingüistica y cultural. Bleichenbacher (2008: 26) afirma que "multilingualism in the text is motivated by the desire to represent a situation of language contact in the story as faithfully as possible". Las aventuras de la famosa familia Simpson se desarrollan en un pueblo llamado Springfield y la serie cuenta con un nutrido grupo de secundarios que reflejan la diversidad social de un gran número de barrios estadounidenses. De hecho, el nombre de Springfield se eligió porque es uno de los nombres de ciudad más comunes de Estados Unidos, por tanto, es otro intento de los creadores por mostrar una realidad reconocible por gran parte del público original. Dore (2016: 122) explica de qué manera las series emplean el lenguaje para reflejar la realidad y cómo recurren a rasgos estereotípicos:

Although they are far from reflecting real interaction stricto sensu, TV series try to recreate a sort of idealised community. In order to do this, the language they use is based on those communicative patterns that are deemed prototypical for a given social group. It is therefore not surprising to find that stereotyped 
language variations are exploited in audiovisual texts to mark differences in social status.

En segundo lugar, la variación lingüística se explota a menudo en los medios audiovisuales como elemento cómico. La inclusión de varios idiomas en un texto audiovisual puede provocar la risa gracias a malentendidos o juegos de palabras interlingüísticos, pero, en ocasiones, es la forma de hablar de un personaje la que pretende ser la causa del humor (recurso que puede relacionarse con sentimientos etnocéntricos). Con respecto a los dialectos, Arampatzis (2012: 67) afirma que constituyen "a fairly popular means of creating humorous sequences".

No obstante, ya sea la intención del guionista añadir un toque cómico, ya sea indicar la nacionalidad de un personaje en favor de la autenticidad, la representación de distintas culturas en los productos audiovisuales suele caer, como ya hemos mencionado, en el uso de estereotipos.

The use and manipulation of language variation to establish character are longestablished practices in storytelling; [...] stage actors used language accent to draw character quickly, building on well-established, preconceived notions associated with specific regional loyalties, ethnic, racial alliances or economic status. This shortcut to characterization means that certain traits need not be laboriously demonstrated by means of a character's history and actions and an examination of motive. (Lippi-Green 2012: 104)

Bleichenbacher (2008) revela en una interesantísima investigación sobre el cine de Hollywood cómo la elección de la nacionalidad de los personajes ha creado y perpetuado peligrosos estereotipos y, también, cómo la aplicación de las estrategias de presencia, evocación o señalización repercute igualmente en la caracterización de los personajes.

Code-switching and interlanguage are typical for negative or laughable minor characters, whereas higher up in the hierarchy, the evil masterminds are more often portrayed as fluent users of standard English [...] characters with pronounced L2 accents, with a preference for code-switching into the replaced language, or with even more obvious instances of interlanguage, are often minor, comical, less powerful, or even downright negative characters. (Bleichenbacher 2008: 83-90)

Además, observamos que, con el fin de conceder una característica destacable a los personajes, se tiende a recurrir a la exageración de los rasgos típicos de la comunidad de la cual provienen. Se persigue, básicamente, que los atributos de esos personajes sean lo suficientemente perceptibles como para que todos los espectadores de la cultura meta identifiquen la diferencia y reconozcan el 
origen del personaje. Steffensen (2012: 510-511) analiza la representación de personajes de Japón y Botsuana en la BBC por medio de acentos y señala lo siguiente:

Accents are used to represent and translate the outside in stereotyping ways that tend towards racialisation and towards actors using generic "Southern African" and "East Asian" accents that bear little resemblance to the actual phonological profile of native speakers of Tswana and Japanese. [...] The accent enacted can tell us that a character speaking in English is Batswana or Japanese, or that the listener should believe so. But what happens when the characterisation is unrecognisable to a Batswana or Japanese person and s/he feels that his/her identity has been misrecognised?

A pesar de que tendremos en cuenta estos aspectos, el objetivo de esta investigación no es analizar si la imitación de los acentos es precisa o si un miembro de esa comunidad se sentiría identificado con el acento que se le adjudica pues, como menciona Steffensen en el caso de la BBC, la intención es que el acento, y por tanto el origen geográfico, sea reconocido por los espectadores. Consideramos que The Simpsons (Matt Groening 1989- ), tanto en VO como en $\mathrm{VD}$, tiene el mismo objetivo.

\section{Introducción al análisis}

El análisis consiste en un estudio descriptivo que compara la versión original de la serie con su versión doblada al español de España. Sin embargo, como es bien sabido, esa versión se obtiene tras las fases de traducción, ajuste y grabación en sala y, por tanto, la versión que se emite en televisión puede distar considerablemente de la propuesta ofrecida por la traductora. Por ello, con el objetivo de dilucidar no solo cómo se doblan los acentos, sino también qué papel juega la traductora en la toma de estas decisiones, hemos querido analizar no solo el producto, sino también parte del proceso. Esto ha sido posible gracias a la información proporcionada por la traductora de la serie para su doblaje en España, María José Aguirre de Cárcer. De este modo, las opciones de traducción analizadas en el corpus pueden explicarse a menudo con las justificaciones de la propia traductora.

El corpus recoge una selección de 32 personajes que presentan un acento que diverge del estándar estadounidense. Es necesario recalcar aquí que el presente artículo no pretende incluir todos los personajes con acento que muestra la serie, pues en The Simpsons aparecen infinidad de secundarios y sus más de 600 capítulos la convierten en una de las producciones más largas de la historia de la televisión. El objetivo de este estudio tampoco es realizar 
un análisis exhaustivo de las características fonéticas y prosódicas de los personajes, pero, a modo de ilustración, incluiremos algunos de los rasgos que caracterizan el acento prototípico de los hablantes de cada variedad diatópica analizada. Cuando esos rasgos coincidan en ambas versiones solo se mencionarán una vez.

Para agrupar los ejemplos se han seguido tres criterios. En cierto modo, los acentos ya clasifican a los interlocutores pues, por un lado, dividen a los hablantes de una lengua en hablantes nativos y no nativos y, por otro, separan a la comunidad de hablantes nativos en diferentes zonas geográficas. Por ello, se ha tenido en cuenta en primer lugar si el acento es extranjero, es decir, si son hablantes del inglés como lengua no nativa (L2), o regional, es decir, si hablan un dialecto del inglés como nativos (L1). Estos dos apartados se subclasifican a su vez teniendo en cuenta el resultado obtenido en la VD: acento extranjero, acento regional o acento estándar. Por último, se han agrupado los personajes que comparten la misma lengua de origen, especificando además su país o zona de procedencia. Solo se ha mencionado el número de capítulo en el caso de secundarios que aparecen solo una o dos veces en la serie.

\section{Análisis del doblaje de los acentos extranjeros y regionales en The Simpsons}

\subsection{Acento extranjero (inglés como L2)}

En este apartado se recogen los personajes de la VO que no hablan inglés como lengua nativa, sino que lo hablan como segunda lengua (L2). Su forma de hablar se caracteriza por un acento procedente de su lengua materna. A continuación, veremos cómo esta situación inicial puede dar pie a tres resultados distintos en el doblaje: español con acento extranjero, español con acento dialectal y español con acento estándar. 
4.1.1. Inglés (L2) con acento extranjero > español (L2) con acento
extranjero

Tabla 1. Personajes doblados al español con acento extranjero

\begin{tabular}{|c|c|}
\hline $\begin{array}{l}\text { CARACTERÍSTICAS DEL ACENTO } \\
\text { EXTRANJERO }\end{array}$ & PERSONAJES \\
\hline $\begin{array}{l}\text { Alemán. VO acento alemán. VD acento } \\
\text { alemán. Sustitución de la vibrante alveolar } \\
\text { simple /r/ por la vibrante alveolar múltiple } \\
\text { /r/. }\end{array}$ & $\begin{array}{l}\text { (1) Fritz. Alemania (capítulo 11, } \\
\text { temporada 3). } \\
\text { (2) Hans. Alemania (capítulo 11, } \\
\text { temporada 3). } \\
\text { (3) Horst. Alemania (capítulo 11, } \\
\text { temporada 3). } \\
\text { (4) Utter Zörker. Alemania. } \\
\text { (5) Hitler. Alemania. } \\
\text { (6) Rainier Wolfcastle. Austria. } \\
\text { (7) Wolfgang Puck. Austria (capítulo 11, } \\
\text { temporada 13). }\end{array}$ \\
\hline $\begin{array}{l}\text { Francés. VO acento francés. VD acento } \\
\text { francés. Acentuación de la última sílaba. } \\
\text { Aspiración de la última letra de la palabra. }\end{array}$ & (8) Cesar. Francia. \\
\hline $\begin{array}{l}\text { Italiano. VO acento italiano. VD acento } \\
\text { italiano. Tendencia a enfatizar las vocales } \\
\text { átonas internas. }\end{array}$ & $\begin{array}{l}\text { (9) Angelo. Italia. } \\
\text { (10) Luigi Risotto. Italia. } \\
\text { (11) Francesca Terwilliger. Italia. }\end{array}$ \\
\hline $\begin{array}{l}\text { Japonés. VO acento asiático. VD acento } \\
\text { asiático. Sustitución de /r/ por } / 1 / \text {. }\end{array}$ & (12) Doctor Sakamoto. Japón. \\
\hline $\begin{array}{l}\text { Ruso. VO acento ruso. VD acento ruso. } \\
\text { Sustitución de la vibrante alveolar simple } \\
\text { /s/ por la vibrante alveolar múltiple /r/. }\end{array}$ & $\begin{array}{l}\text { (13) Zhenya. Rusia (capítulo 20, } \\
\text { temporada 24). } \\
\text { (14) Slava. Rusia (capítulo 20, temporada } \\
\text { 24). }\end{array}$ \\
\hline
\end{tabular}

Esta situación, en la que los hablantes del original no son nativos de la lengua origen (LO) y en que la lengua nativa del personaje no coincide con la lengua meta (LM), no presenta grandes obstáculos en la traducción de la variedad geográfica en forma de acento pues, si queremos mantener el recurso, basta con reflejar la pronunciación típica de esa comunidad al hablar en castellano. Así, en la VD los personajes hablan un español caracterizado por un acento extranjero; esto es, todos hablan un español como segunda lengua y, por tanto, incluyen rasgos fonológicos de su lengua materna. Esta solución se podría denominar mantenimiento, pues se mantiene el mismo recurso empleado en la versión original. Aguirre de Cárcer afirma que, para indicar que un personaje debe poseer un acento que diverja del estándar, escribe el texto que el actor de doblaje debe interpretar e incluye una nota en la que indica el acento que debe añadirle pues, según explica, especificar el acento del personaje resulta 
más claro que escribir cómo debería pronunciarse. Por ejemplo, si uno de los personajes (1) a (7) dijera la frase "Tengo mucha hambre", la traductora lo indicaría del siguiente modo: [Con acento alemán] Tengo mucha hambre; en lugar de recurrir a símbolos fonéticos o escribir la línea de diálogo tal y como lo pronunciaría una persona con un acento alemán marcado. Por tanto, la representación del extranjero dependerá en gran medida de la idea que la comunidad meta tenga de cómo habla su lengua esa comunidad y, sobre todo, de la interpretación de los actores y las indicaciones del director de doblaje.

Cabe remarcar que cuando el idioma materno de los personajes es cercano a países occidentales (España o EE. UU.) — como en el caso del italiano, el francés o el alemán (ejemplos del 1 al 11)—es más fácil que el espectador lo reconozca. Sin embargo, cuando se trata de personajes procedentes de países más exóticos para los espectadores observamos que se utiliza, tanto en la VO como en la VD, un acento que se vincula no a un idioma concreto, sino a una familia de lenguas. Por ejemplo, si el espectador solo tuviera acceso a la pista de audio, podría creer que el personaje número 12 es chino en lugar de japonés porque el acento empleado se vincula de manera general con las lenguas asiáticas.

Aparte de los cambios en la pronunciación con respecto a la lengua estándar, observamos que se recurre con frecuencia a la señalización, es decir, los propios personajes mencionan de forma explícita su lengua, religión o procedencia. Además, con el fin de subrayar que el personaje no está hablando su lengua nativa, se suelen introducir, tanto en la versión original como en la doblada, palabras o expresiones en su primera lengua. Así, los personajes alemanes pronuncian palabras como Nein o Guten Morgen. A este respecto, Bleichenbacher (2008: 83) se preguntaba en su investigación sobre el multilingüismo en Hollywood "to what extent the use of interlanguage features for evocation is paralleled by a depiction of non-English L1 speakers as inept L2 users of English (and other languages)?".

La forma de hablar de Hans (2) en la versión doblada resulta especialmente llamativa precisamente por aumentar considerablemente la "ineptitud" del personaje. En el doblaje al español no solo introduce palabras en su lengua materna, sino que además comete errores tanto en la lengua que no domina (No problema) como en la que sería su lengua materna (la pronunciación de los números en alemán es incorrecta). Quizá este fallo se deba a que el guion doblado no incluía una lista de pronunciación y el actor de doblaje no tenía conocimientos de alemán.

El mantenimiento es una solución muy eficaz porque conserva tanto el recurso empleado en la versión original como su función, pero dejaría de ser 
viable si el acento proviniera precisamente del español, es decir, de la LM, tal y como ocurre en el siguiente apartado.

\subsubsection{Inglés (L2) con acento extranjero > español (L1) con acento regional}

Tabla 2. Personajes doblados al español con acento regional

\begin{tabular}{|l|l|}
\hline \multicolumn{1}{|c|}{$\begin{array}{c}\text { CARACTERÍSTICAS DEL ACENTO } \\
\text { EXTRANJERO }\end{array}$} & \multicolumn{1}{c|}{ PERSONAJES } \\
\hline $\begin{array}{l}\text { Español. VO acento hispano. } \\
\text { Pronunciación de [ } \theta \text { ] a final de palabra } \\
\text { como [t]. VD español de Argentina. } \\
\text { Yeísmo rehilado. }\end{array}$ & $\begin{array}{l}\text { (15) Doctor Nick Riviera. VO: } \\
\text { Latinoamérica. VD: Argentina. } \\
\text { (16) Personaje sin nombre (capítulo 4, } \\
\text { temporada 14). VO: Latinoamérica. VD: } \\
\text { Argentina. }\end{array}$ \\
\hline
\end{tabular}

La VO de los ejemplos 15 y 16 muestra a dos personajes que hablan inglés como L2. Aunque no resulta sencillo concretar su país de procedencia, el acento empleado evidencia que son de origen hispanohablante. Por su parte, la VD, muestra a dos personajes cuyo español, además de ser su L1, tiene un marcado acento argentino. Por tanto, en estos dos ejemplos se propone como equivalente de un acento de un personaje no nativo una variedad dialectal de la lengua meta. Este cambio se debe a que la Ll del personaje de la VO coincide con la lengua de la VD, lo que hace que la técnica de mantenimiento comentada en el apartado anterior sea inviable. A pesar de que esta solución aparece recogida con diversos nombres en las obras de otros autores como Reutner, que la denomina "equivalencia indirecta mediante las variedades de la lengua meta" (2013: 155), o García Luque, que habla de "presencia de un acento propio de la LM en la LO" (2016), nosotras proponemos el término dialectización por su brevedad y transparencia. Es importante subrayar que durante el proceso de doblaje se ha tomado una decisión sobre la procedencia de los personajes y, probablemente, se ha elegido en base al reconocimiento que pueda tener para el espectador del texto meta. La propia traductora corrobora esta hipótesis en el caso de Nick, pues comenta que fue el director de doblaje quien decidió otorgar un acento argentino al doctor con el fin de hacerlo más reconocible y cómico. Sin embargo, sería importante recalcar de nuevo la perpetuación de estereotipos. Según Piñeiro (2004), la caracterización de los personajes argentinos por medio de particularidades discursivas como la fluidez verbal ha "propiciado la consolidación del estereotipo de argentino en determinadas profesiones como la de psicoanalista (y determinadas variantes como psicólogo, psiquiatra, terapeuta...)". 
4.1.3. Inglés (L2) con acento extranjero > español (L2) con acento estándar

Tabla 3. Personajes doblados al español con acento estándar

\begin{tabular}{|l|l|}
\hline \multicolumn{1}{|c|}{$\begin{array}{c}\text { CARACTERÍSTICAS DEL ACENTO } \\
\text { EXTRANJERO }\end{array}$} & \multicolumn{1}{|c|}{ PERSONAJES } \\
\hline $\begin{array}{l}\text { Albanés. VO acento albanés. Sustitución } \\
\text { de la vibrante alveolar simple /r/ por la } \\
\text { vibrante alveolar múltiple /r/. VD español } \\
\text { estándar. }\end{array}$ & (17) Adil Hoxha. Albania. \\
\hline
\end{tabular}

El caso de Adil Hoxha resulta interesante porque, a pesar de que se podría haber acudido a un mantenimiento, la VD se decanta por una estandarización. Una hipótesis que explicaría esta decisión es que el equipo de doblaje no supiera cómo imitar a un albanés y optara por estandarizar el acento, pero esta decisión resulta incoherente con la pista de audio, ya que el director Skinner presenta a Adil de la siguiente manera: "Puede que su acento os parezca peculiar, y algún aspecto de su cultura os parecerá absurdo, o incluso ofensivo, pero yo os ruego que le concedáis el beneficio de la duda, única manera de intentar comprender a estos nativos retrógrados que nos llegan desde el tercer mundo". Cuando le pedimos a María José Aguirre de Cárcer que nos justificara esta estandarización del acento extranjero, se quedó muy sorprendida porque, en su opinión, si el niño tiene acento en la VO, también debería tenerlo en la VD. Cuando vio el capítulo doblado, nos comentó que tenía que deberse a un error en el proceso de doblaje.

\subsection{Acento regional (inglés como L1)}

Los personajes que presentan una variedad dialectal del inglés como lengua materna (L1) se trasvasan en la VD de tres modos: por medio de un acento extranjero, regional o estándar. A pesar de que estas soluciones son las mismas que las encontradas para el doblaje del acento extranjero, observaremos durante el análisis que las causas y consecuencias de dichas decisiones de traducción difieren con respecto a las comentadas en el apartado 4.1. 
4.2.1. Inglés (L1) con acento regional > español (L2) con acento extranjero

Tabla 4. Personajes doblados al español con acento extranjero

\begin{tabular}{|c|c|}
\hline $\begin{array}{l}\text { CARACTERÍSTICAS DEL ACENTO } \\
\text { REGIONAL }\end{array}$ & PERSONAJES \\
\hline $\begin{array}{l}\text { Inglés. VO acento británico. Linking r: la } \\
\text { /r/ solo se pronuncia cuando va seguida } \\
\text { de vocal. VD acento británico. Adición del } \\
\text { sonido }[u] \text { a las palabras acabadas en /o/. } \\
\text { Pronunciación alveolar de los fonemas /d/, } \\
\text { /t/ y /r/. }\end{array}$ & $\begin{array}{l}\text { (18) Eliza Simpson (capítulo 1, temporada } \\
\text { 16). Inglaterra. } \\
\text { (19) Dr. Bartley (capítulo 1, temporada } \\
\text { 16). Inglaterra. } \\
\text { (20) Homer (capítulo 1, temporada 16). } \\
\text { Inglaterra. } \\
\text { (21) Jeff Albertson, vendedor de cómics } \\
\text { (capítulo 1, temporada 16). Inglaterra. } \\
\text { (22) Secundaria sin nombre. Prostituta } \\
\text { asesinada (capítulo 1, temporada 16). } \\
\text { Inglaterra. }\end{array}$ \\
\hline $\begin{array}{l}\text { Inglés. VO acento del sur de Asia. } \\
\text { Pronunciación del diptongo [j] como [dz]. } \\
\text { VD acento del sur de Asia. Sustitución } \\
\text { de la vibrante alveolar múltiple /r/ por la } \\
\text { vibrante alveolar simple /r/. }\end{array}$ & $\begin{array}{l}\text { (23) Apu. India. } \\
\text { (24) Manjula. India. }\end{array}$ \\
\hline
\end{tabular}

Los personajes 18-24 se caracterizan en la VO por el uso de un acento propio de un dialecto del inglés. Aunque en principio la versión doblada está optando por mantener el acento tal y como veíamos en el apartado 4.1.1, hay un cambio sustancial, ya que se está añadiendo un acento extranjero a un personaje que es nativo en la $\mathrm{VO}$, es decir, se les convierte en personajes que hablan el idioma como no nativos (L2). Steffensen (2012: 514) subraya lo que implica el uso de un acento no nativo: "Non-native accent signifies that the bearer of the accent is an outsider, masters the language incompletely and is therefore often considered culturally inferior to native speakers".

Para subrayar el cambio que supone, consideramos que esta solución se podría denominar extranjerización.

Los primeros cinco personajes de este apartado aparecen en una de las historias que recoge el primer capítulo de la temporada 16, que se desarrolla en el Londres de finales del siglo XIX. En la versión original, los personajes de Springfield se convierten en personajes londinenses y una de las manifestaciones de esta transformación es el cambio de acento. Sin embargo, como veremos en el apartado 4.2.3, no todos los personajes británicos que aparecen en ese capítulo presentan un acento de Inglaterra en la versión doblada. Con respecto a este mantenimiento del acento británico, cabría destacar dos aspectos interesantes. En primer lugar, el hecho de que los personajes dejen 
de ser nativos en la VD permite a la traducción recurrir a la estrategia de codeswitching, de modo que, por ejemplo, Eliza incluye en su discurso una palabra de su lengua nativa (well). Por otro lado, resulta curioso que el acento no es igual de marcado en todos los personajes. Eliza presenta un acento mucho más exagerado que el del Dr. Bartley.

Además de incluir el acento británico, la procedencia de los personajes se subraya por medio del registro. Aguirre de Cárcer afirma que para traducir el inglés de Inglaterra opta por un español más correcto y arcaico. De esta manera se refuerzan estereotipos asociados a los británicos:

British English is largely associated with a high social class, giving way to two different sets of stereotypes: positive ones, such as elegance, sophistication, good manners and intellectuality, or negative ones, such as arrogance, snobbishness and cold-heartedness (Arampatzis 2012: 79-80)

Los personajes de Apu y Manjula merecen una atención especial pues, si bien son hablantes nativos de inglés, es muy probable que en la serie no se concibieran como tales debido a una confusión muy extendida que Shilpa (2013: 318) aclara del siguiente modo:

Although perceived and received as a "foreign accent" in American culture, Indian English is, in fact, a native form of speaking in India, where the two national languages are English and Hindi. [...] So although it would be more appropriate to think of Indian English as an inflected version of English that more closely resembles British English, American culture perceives Indian Americans as talking with an accent of a nonnative speaker.

Además, volvemos a observar la tendencia a utilizar acentos genéricos más relacionados con familias de idiomas que con idiomas en sí, y que ya hemos señalado en personajes como el Dr. Sakamoto (12). Shilpa (2013: 41) asegura que el acento de Apu no es exactamente indio, lo que refuerza los estereotipos:

Apu is identified as Indian in the series, [...] but his accent stands in for a more general South Asian accent. Despite the inclusion of Apu in a social satire of American culture, his signature voice, I argue, is an example of a racialised performance of South Asians in the United States that reinforces rather than challenges stereotypes of South Asian Americans and, more generally, Asian Americans in American media.

La explotación de los clichés no solo se encuentra en el uso del acento, sino también en la imagen y el guion. Estas características de los personajes indios persisten en la VD y afianzan estereotipos arraigados tanto en la sociedad norteamericana como en la española. Manjula y Apu activan el estereotipo de la 
cultura machista en la que el hombre ostenta un puesto de trabajo, mientras la mujer se queda en casa cuidando de sus numerosos hijos.

Además, aunque los acentos de estos personajes son claramente reconocibles para un espectador hispanohablante, su procedencia se refuerza con la aparición de elementos culturales. Por ejemplo, la versión doblada añade la expresión iPor los brazos de Shiva!

\subsubsection{Inglés (L1) con acento regional > español (L1) con acento regional}

Este subapartado recoge dos ejemplos en los que una variedad regional de la LO se ha sustituido por un acento regional de la LM, es decir, se propone un dialecto de la LM como equivalente del que aparece en la VO.

Tabla 5. Personajes doblados al español con acento regional

\begin{tabular}{|l|l|}
\hline \multicolumn{1}{|c|}{$\begin{array}{c}\text { CARACTERÍSTICAS DEL ACENTO } \\
\text { REGIONAL }\end{array}$} & \multicolumn{1}{|c|}{ PERSONAJES } \\
\hline $\begin{array}{l}\text { Inglés. VO acento del sur de Estados } \\
\text { Unidos. Sustitución de /y/ a final de } \\
\text { palabra por [n]. VD acento andaluz (?). } \\
\text { Caída de la /s/ a final de palabra y de la /d/ } \\
\text { intervocálica ("to" en lugar de "todo" o } \\
\text { "pue" en lugar de "puede"). }\end{array}$ & \\
\hline $\begin{array}{l}\text { Inglés. VO acento afroamericano. } \\
\text { Eliminación de la /r/ postvocálica. VD } \\
\text { acento cubano. Caída de la /s/ a final de } \\
\text { palabra. Pronunciación del sonido [ } \theta \text { ] } \\
\text { como [s]. }\end{array}$ & $\begin{array}{l}\text { (26) Cómico afroamericano. (Capítulo } 13 \\
\text { de la temporada 5) }\end{array}$ \\
\hline
\end{tabular}

La sustitución de un dialecto de LO por uno de la LM es una solución polémica que muchos autores condenan. Slobodník (1970: 142) afirma lo siguiente:

Tous ces auteurs [Georges Mounin, Fedorov, Güttinger, Levý] sont arrivés à des conclusions identiques : l'emploi du dialecte de la langue de but pour rendre les éléments des dialectes de la langue de départ serait erroné et donnerait à l'aspect sémantique ("signifié") de l'original quelque chose d'absurde et d'indésirablement comique.

Precisamente por ello, en las comedias parece una solución más aceptada que en otros géneros, pero el quid de la cuestión es que en estos casos el doblaje no mantiene un acento que ya aparece en el original, sino que elige uno. Además, el uso de este acento suele presentar una intención humorística. 
Con respecto a la familia Spuckler, el acento empleado en la versión original es el "Southern American English", que consiste en un "one-size-fits-all accent when attempting to 'sound Southern"” (Lippi-Green 2012: 221). Según la autora (2012: 223), una de las características principales del estereotipo del sureño es la ignorancia.

Aguirre de Cárcer asegura que su intención era crear unos personajes "paletos" como los del original, pero que en ningún momento pretendía introducir la identidad andaluza. De hecho, muchos rasgos de su forma de hablar se asociarían más bien a un sociolecto (como la caída de la /d/ intervocálica), pero otras peculiaridades como la caída de la /s/ final sí se relacionarían con acentos geográficos. Se sustituye así un modo de hablar que se relaciona con la incultura por otro que se asocia a estereotipos similares (Teruel y Fernández, 2005). De este modo, el doblaje introduce un cliché que no aparecía en el original y perpetúa en este caso concreto el tópico del atraso de la sociedad andaluza. Un tópico que se ha explotado a menudo a lo largo de la historia del cine y la televisión en nuestro país tanto en la producción propia (Teruel y Fernández, 2005) como en la ajena. Con respecto a las versiones dobladas, basten como ejemplo Hotel Transilvania (Genndy Tartakovsky 2012), Toy Story 3 (Lee Unkrich 2010), Puss in Boots (El gato con botas, Chris Miller 2011) o Around the World in Eighty Days (La vuelta al mundo en 80 días, Michael Anderson 1956).

En el caso del personaje 26, es importante destacar que la elección del acento está condicionada por las restricciones visuales. La imagen muestra un personaje de piel oscura, lo que obliga a seleccionar un dialecto del español que resulte verosímil que hablen personas de raza negra. Por tanto, teniendo en cuenta el canal visual, resultará más verosímil que el personaje de la versión doblada sea portorriqueño, dominicano o cubano, en lugar de argentino o chileno.

\subsubsection{Inglés ( $\mathrm{Ll}$ ) con acento regional > español con acento estándar}

En este apartado se recogen los ejemplos de personajes con acento regional en la versión original que muestran un acento estándar en la versión doblada. 
Tabla 6. Personajes doblados al español con acento estándar

\begin{tabular}{|c|c|}
\hline $\begin{array}{l}\text { CARACTERÍSTICAS DEL ACENTO } \\
\text { REGIONAL }\end{array}$ & PERSONAJES \\
\hline $\begin{array}{l}\text { Inglés. VO acento de Inglaterra. Linking r: } \\
\text { la /r/ solo se pronuncia cuando va seguida } \\
\text { de vocal. VD acento estándar. }\end{array}$ & $\begin{array}{l}\text { (27) Henry VIII / Enrique VIII (capítulo } \\
\text { 11, temporada 15). Inglaterra. } \\
\text { (28) Elton John. Inglaterra. } \\
\text { (29) Chief Wiggum / Jefe Wiggum } \\
\text { (capítulo 1, temporada 16). Inglaterra. } \\
\text { (30) Marge (capítulo 1, temporada 16). } \\
\text { Inglaterra. } \\
\text { (31) Selma (capítulo 1, temporada 16). } \\
\text { Inglaterra. }\end{array}$ \\
\hline $\begin{array}{l}\text { Inglés. VO acento escocés. Pronunciación } \\
\text { rótica: énfasis de la /r/. VD acento } \\
\text { estándar. }\end{array}$ & (32) Willie McDougal. Escocia. \\
\hline
\end{tabular}

Para el espectador de la serie original la variedad diatópica empleada revela que estos personajes provienen del Reino Unido, ya sea de Inglaterra o de Escocia. Ese acento que los distingue del resto de los personajes desaparece en la VD. Aun así, en algunos casos el espectador meta puede reconocer igualmente su procedencia gracias a que encarnan a personas reales mundialmente conocidas (ejemplos 27 y 28) o gracias a la señalización ${ }^{2}$.

Además, en el caso de los personajes de Inglaterra, como ya hemos mencionado anteriormente, Aguirre de Cárcer los caracteriza con un lenguaje más refinado, correcto y arcaico. Por tanto, se podría decir que se ha optado por una formalización del registro. No se trata de un gran cambio con respecto al original, que ya emplea un registro formal, pero sí es latente la intención de la traducción de subrayar esa formalidad y, por tanto, el estereotipo asociado con los ingleses en la cultura meta.

Por otro lado, la traducción de los personajes 29, 30 y 31 resulta llamativa porque, como hemos avanzado en el apartado 4.2.1, aparecen en un capítulo con otros personajes británicos a los que sí se les representa en la versión doblada por medio de acento extranjero (ejemplos 18-22). A este respecto, Lippi-Green (2012: 109) observa una falta de consistencia en el uso de los acentos en los productos audiovisuales originales y advierte que es frecuente que la evocación se use solo con algunos personajes y no con todos los que deberían tener un acento determinado siguiendo la lógica:

2. Por ejemplo, el jefe Wiggum (29) afirma en el capítulo que le transforma en británico: Los ingleses comemos lo que sea. 
If a French accent is meant to remind viewers that the story is taking place in France, then logic would require that all the characters in that story speak with a French accent. But this is not the case in animated or live action; for the most part, in movies set outside English-speaking countries only a few actors will contrive the accent of that country.

En el caso del doblaje, García Luque (2007: 452) señala que

el tratamiento de los acentos recibe una atención especial cuando alguno de los protagonistas se distingue del resto por su peculiar forma de hablar, pero aquellos casos en que todos los personajes o la gran mayoría de ellos habla con un acento determinado, este factor es obviado en la traducción.

Una posible hipótesis es que se decide extranjerizar solo a los protagonistas de la historia y estandarizar a los secundarios. Aguirre de Cárcer confirmó nuestra suposición explicándonos que utilizar el acento británico durante toda la historia resultaría demasiado recargado. No obstante, no parece un criterio que se haya aplicado con rigor, ya que observamos que personajes con pocas líneas como Jeff Albertson (21) o la primera prostituta asesinada (22) sí tienen acento, mientras que otros como el jefe Wiggum (29), que es sin duda uno de los protagonistas de la historia, no.

Por su parte, Lippi-Green (2012: 109) señala otras causas para la decisión de incluir o no acentos:

The decision about which actors will try to sound French, for example, is not random, but follows logically from the dominant stereotypes (or in some cases, from the actor's native language). Consider Disney's Beauty and the Beast (Trousdale and Wise 1991, directors) set in France. All of the major characters speak English with American accents with three exceptions: the sexy chamber maid, the amorous butler, and a temperamental cook are voiced by actors contriving French accents.

Con respecto al personaje 32, cabe señalar que las alusiones a su origen son constantes en el canal visual. Willie es un pelirrojo que suele llevar kilt e, incluso, tocar la gaita. Por tanto, las restricciones visuales suponen tanto una limitación como una ayuda para el trasvase de información. Por un lado, el hecho de que la imagen remarque con tal frecuencia su nacionalidad logra que para el espectador meta también resulte evidente que el personaje es escocés y, por tanto, la traductora no tendrá necesidad de incluir dicha información en el diálogo. Como afirmaba Martínez Sierra (curiosamente en una investigación sobre la misma serie publicada en 2008), la comprensión del espectador meta se ve favorecida por la gran aportación semiótica que ofrecen los diversos códigos emitidos de forma simultánea. Por otro lado, la imagen restringe las 
posibilidades de la traductora, pues queda descartado ${ }^{3}$ el uso de otros dialectos (técnica analizada en el apartado 4.2.2).

Para compensar la pérdida de la variedad lingüística diatópica, la VD recurre a dos particularidades paralingüísticas. La primera es la cualidad de la voz y la segunda, la manera de hablar (Reutner 2013: 154-155), que engloba la intensidad de la voz, la velocidad y la claridad. Así en la VD la voz de Willie se caracteriza por un timbre agudo y una voz fuerte y algo ronca, rasgo que, según Reutner connota una mente sencilla. En cuanto a su modo de hablar, se opta por un registro vulgar.

La informalización o vulgarización del registro es una técnica frecuente en $\mathrm{TAV}^{4}$, pero es importante puntualizar que el hecho de que un hablante tenga determinado acento no implica que su registro sea informal. De hecho, la informalización o vulgarización del registro está íntimamente vinculada con la sociolectización, que consistiría en proponer como equivalente de un acento un sociolecto, pues al rebajar el registro solemos rebajar también la clase social. Así, traducir de manera sistemática determinados dialectos por un registro coloquial puede perpetuar estereotipos relacionados con la falta de formación de determinados grupos sociales.

Tras analizar los ejemplos de este apartado podría destacarse que, incluso cuando se opta por estandarizar el acento que caracteriza a los personajes de la $\mathrm{VO}$, la traductora puede recurrir a otros elementos para remarcar las diferencias como el registro, la cualidad de la voz o las maneras de hablar.

\section{Resultados del análisis}

Tal como se ha podido apreciar tras el análisis del doblaje de 32 personajes caracterizados en The Simpsons por el acento, existen formas muy diversas de enfrentarse a su doblaje. Para elegir entre esas posibilidades de traducción son determinantes factores lingüísticos como la coincidencia de la Ll del personaje con la lengua de la VD o el grado de dificultad para representar un acento determinado en la VD, pero, también, aspectos extralingüísticos como la intención de la variante en la VO, las restricciones visuales, las características

3. Resulta curioso que, a pesar de la carga semántica aportada por el canal visual, la versión doblada al italiano haya sustituido el acento escocés de Willie por el sardo (Dore 2009: 142).

4. Ranzato (2010: 112) afirma que el doblaje italiano tiende a sustituir los rasgos dialectales por expresiones y léxico incorrecto o informal que no marcan al personaje geográficamente. El análisis de Lomeña (2009) sobre la traducción al italiano y el francés de la película Mujeres al borde de un ataque de nervios ilustra esta situación. 
que se intentan destacar del personaje o, incluso, el número de personajes con acento que aparecen en un episodio determinado.

En primer lugar, se debe destacar que la aparición de acentos en la serie no es circunstancial, sino que es una decisión meditada, por tanto, la traducción se esfuerza por trasvasar el acento o, si esto no es posible, los rasgos que se están intentando destacar en la VO. También es necesario recordar que la adscripción de los personajes a diversos territorios no se hace exclusivamente por medio del acento, sino que tanto la versión original como la doblada están repletas de pistas: nombres de personajes (Luigi Risotto, Sakamoto o Fritz), referencias culturales (ravioli, Shiva, Putin), deformación del léxico (masticare), code-switches (Eins, zwei, drei; Well) o señalización (Nein, somos alemanes. Él, alemán occidental; yo, alemán oriental).

Si el acento proviene de una lengua distinta de la L2, el trasvase no ofrece grandes obstáculos y se recurre al mantenimiento (1-14); con la excepción del personaje albanés (17). Dore (2016: 132) observa esta tendencia también en el doblaje al italiano. La autora afirma que, cuando el acento es extranjero, el procedimiento más habitual es mantenerlo en la versión doblada para que el personaje tenga un acento marcado en la lengua meta.

Es necesario puntualizar aquí que en ocasiones se percibe un acento más marcado en la VD que en la VO. Esta tendencia a exagerar el acento de los extranjeros no es exclusiva de Los Simpson, pues García Luque (2007: 450) también lo identifica en el análisis de otros productos audiovisuales: "Digamos que lo que el original simplemente apunta, el doblaje lo subraya notablemente". Creemos que esta exageración tiene dos finalidades. En primer lugar, que el espectador reconozca claramente una pronunciación que diverge del estándar y que le remita a un origen concreto o, al menos, a identificar a un personaje como no nativo. En segundo lugar, potenciar la intención humorística.

Sin embargo, cuando la lengua de la que proviene el acento coincide con la lengua meta la opción de añadir un acento extranjero queda descartada. Por esta razón, en el corpus se recurre a la dialectización. Esta solución logra mantener el acento y la procedencia del personaje de la VO, pero supone dos cambios en su caracterización: primero, el personaje se convierte en un hablante nativo y, segundo, su país de origen se especifica. Los personajes (15) y (16) son de Latinoamérica, pero en la versión doblada se concreta su país de origen: Argentina.

Los acentos que provienen de un dialecto de la LO hacen referencia a una realidad delimitada dentro de esta y cuyas características son difícilmente reconocibles por el espectador meta o difícilmente plasmables en la LM. 
Observamos que en estos casos la traductora ha recurrido a tres soluciones: la extranjerización, la sustitución y la compensación.

La extranjerización es en realidad una variante del mantenimiento, ya que lo que hace la traductora es mantener el acento que aparece en la VO. Lo que cambia, sin embargo, es que en la VO el personaje es nativo, mientras que en la VD el personaje es extranjero (personajes 19-24).

La sustitución consiste en proponer como equivalente de un acento regional de la LO, un acento regional de la LM. Por tanto, la traducción cambia la procedencia del personaje. Así, en el análisis veíamos, por ejemplo, cómo un personaje afroamericano se convertía en cubano por arte del doblaje (26).

La neutralización total consistiría en emplear un acento estándar y no utilizar ningún recurso de compensación (17). Esta técnica podría justificarse en casos en los que el acento fuera una característica circunstancial del personaje.

En el corpus analizado, la desaparición del acento prácticamente siempre viene acompañada de algún recurso adicional que tiene como objetivo compensar la pérdida, por ejemplo, elevar el registro (personajes británicos), rebajarlo (32) o modificar las características de la voz (32).

Con respecto a la traducción de los estereotipos, observamos que, por lo general, la VD se limita a mantener los que ya aparecen en la VO. Sin embargo, hay que recalcar algunos cambios que pueden fortalecerlos como la introducción de errores o el uso de un acento más marcado. Además, la VD añade estereotipos no incluidos en la VO cuando elige un acento, como comentamos, por ejemplo, en el caso del psicólogo argentino (15).

\section{Conclusiones}

"Yo no hablo como Apu de Los Simpson". Raj, capítulo 08 de la primera temporada de la serie The Big Bang Theory.

Por lo general, los acentos extranjeros que escuchamos en los productos audiovisuales de ficción - tanto en versiones originales como dobladas- son representaciones que la cultura original y meta respectivamente se hacen de las divergencias fonológicas originadas por lenguas extranjeras y dialectos; es decir, los personajes franceses aparecen representados en la VO tal y como los imitaría un estadounidense y, en la VD, tal y como los imitaría un español. El objetivo no es, pues, una representación realista, sino que el espectador relacione al personaje con una lengua distinta de la Ll. Además, observamos que esos acentos extranjeros pueden relacionarse con idiomas concretos o con familias de lenguas. Por ejemplo, los personajes franceses tienen acento francés, pero los japoneses tienen acento "asiático". Se entiende que esta diferenciación 
se debe a los conocimientos del espectador meta, que reconocerá fácilmente el origen de un personaje cuando su país de procedencia sea próximo o conocido en la cultura meta, pero que encontrará más dificultades a la hora de distinguir entre países de zonas más alejadas y desconocidas como Asia, Oriente Medio o los países eslavos. Por otro lado, exagerar el acento garantiza el reconocimiento de su origen por parte del espectador, pero representar a los extranjeros con un acento marcado que suele aderezarse con dificultades de comprensión o errores gramaticales y semánticos no deja de ser una visión etnocéntrica.

Una de las conclusiones del análisis es que a la hora de elegir la técnica de traducción hay dos factores determinantes: en primer lugar, la intención del doblaje y, en segundo lugar, el tipo de acento susceptible de ser transvasado y su relación con la LM. En el siguiente organigrama, que podría suponer una base para una propuesta de análisis del doblaje de los acentos, se intenta resumir cómo estos factores determinan la técnica de traducción empleada.

Figura 1. Principales factores que influyen en la elección de técnicas de traducción

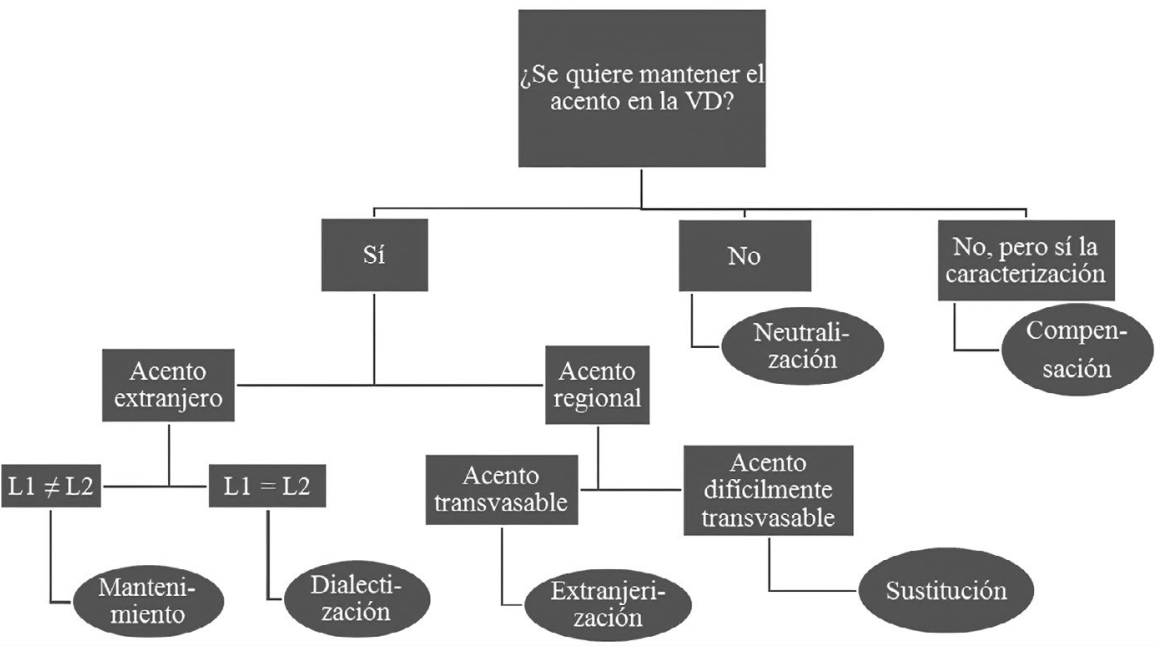

Por último, presentamos una tabla que resume las consecuencias que tienen el uso de estas seis técnicas de traducción: 
Tabla 7. Técnicas de traducción para el doblaje de acentos y sus consecuencias con respecto al acento y la caracterización

\begin{tabular}{|l|c|c|c|l|}
\hline \multicolumn{1}{|c|}{ TÉCNICA } & ACENTO & $\begin{array}{l}\text { ACENTO } \\
\text { VO Y VD }\end{array}$ & $\begin{array}{c}\text { CARACTE- } \\
\text { RIZACIÓN }\end{array}$ & \multicolumn{1}{|c|}{ COMENTARIOS } \\
\hline Mantenimiento & Sí & $=$ & $=$ & \\
\hline Dialectización & Sí & $\neq$ & $\neq$ & $\begin{array}{l}\text { El personaje extranjero } \\
\text { pasa a ser nativo + se } \\
\text { especifica su país de } \\
\text { origen. }\end{array}$ \\
\hline Extranjerización & Sí & $=$ & $\neq$ & $\begin{array}{l}\text { El personaje nativo pasa } \\
\text { a ser extranjero. }\end{array}$ \\
\hline Sustitución & Sí & $\neq$ & $\neq$ & $\begin{array}{l}\text { Se modifica la } \\
\text { procedencia del } \\
\text { personaje. }\end{array}$ \\
\hline Compensación & No & $\mathrm{X}$ & $?$ & $\begin{array}{l}\text { Se intentan trasvasar } \\
\text { rasgos del personaje y } \\
\text { compensar la pérdida } \\
\text { del acento por medio del } \\
\text { registro, la cualidad de } \\
\text { la voz y las maneras de } \\
\text { hablar. }\end{array}$ \\
\hline Neutralización & No & $\mathrm{X}$ & $\neq$ & $\begin{array}{l}\text { El personaje pasa a } \\
\text { hablar un español } \\
\text { estándar. Se neutraliza la } \\
\text { diferencia. }\end{array}$ \\
\hline
\end{tabular}

Este artículo es una modesta incursión a un objeto de estudio poco explorado en el ámbito académico, por lo que sería interesante en el futuro profundizar en algunas de las cuestiones abordadas en la presente investigación, como la búsqueda de otras soluciones factibles para este problema o la asociación entre acentos y estereotipos. A este respecto, se podría realizar un estudio interdisciplinar sobre la representación de la identidad en The Simpsons, pues la serie basa parte de su humor en la explotación de estereotipos, pero también es capaz de cuestionarlos o subvertirlos, tal y como veíamos en las intervenciones de Skinner (apartado 4.1.3) o Slava (línea de diálogo con la que comenzaba este artículo). Igualmente, si bien el presente análisis se ha centrado en el resultado final del doblaje y la intervención de la traductora en él, ha quedado patente que las decisiones sobre los acentos las toman a menudo otros profesionales a lo largo del proceso, por lo que resultaría de gran interés una futura línea de investigación en la que se analizaran en qué medida y de qué modo intervienen cada uno de los eslabones de la cadena en el resultado final. Esto permitiría contrastar hipótesis que hemos lanzado en el estudio, como el caso de la 
estandarización del acento albanés. Además, esta investigación nos ha generado multitud de interrogantes sobre los que sería interesante seguir indagando, como la traducción de aspectos fonológicos relacionados con la clase social o los idiolectos, o la repercusión del género con respecto a la toma de decisiones y a la aceptabilidad del público: ¿recibiría el público con el mismo entusiasmo algunas de las soluciones aportadas en Los Simpson, si no fuera una serie de animación o una comedia? A este respecto podría ser interesante profundizar a partir de la investigación de Pym (2000) y cruzar datos con estudios de ficciones de carne y hueso (como el de Dore 2016) e incluso películas de no ficción.

\section{Bibliografía}

ARampatzis, Christos. (2012) "Dialects at the Service of Humour within the American Sitcom: A Challenge for the Dubbing Translator." En: Ermida, Isabel $\&$ Jan Chovanec (eds.) 2012. Language and Humour in the Media. Newcastleupon-Tyne: Cambridge Scholars Publishing, pp. 67-81.

BleICHENBACHER, Lukas. (2008) Multilingualism in the Movies. Tubinga: Francke Verlag.

Corrius, Montse \& Patrick ZABAlBEASCOA. (2011) "Language Variation in Source Texts and Their Translations: The Case of L3 in Film Translation." Target 23: 1 , pp 113-130.

De Higes-Andino, Irene; Ana María Prats-Rodriguez; Juan José Martínez SIERRA \& Frederic CHAUME. (2013) "Subtitling Language Diversity in Spanish Immigration Films." Meta: journal des traducteurs 58: 1, pp. 134-145.

DORE, Margherita. (2009) "Target Language Influences over Source Texts: A Novel Dubbing Approach in The Simpsons, First Series." En: Federici, Federico M. (ed.) 2009. Translating Regionalised Voices in Audiovisuals. Roma: Aracne, pp. 137-156.

DORE, Margherita. (2016) "The Italian Dubbing of Dialects, Accents and Slang in the British Dark Comedy Drama Misfits." Status Quaestionis 11, pp. 122151. Versión electrónica: <http://statusquaestionis.uniromal.it/index.php/ statusquaestionis/article/view/13835/13602>

GarCía LuQue, Francisca. (2007) "El problema de los acentos en el doblaje." En: Balbuena Torezano, M. ${ }^{a}$ del Carmen \& Ángeles García Calderón (eds.) 2007. Traducción y mediación cultural. Reflexiones interdisciplinares. Granada: Atrio, pp. 441-454.

GARCÍA LUQUE, Francisca. (2016) "Los acentos y otros recursos en el cine de animación." En: Comunicación en formato póster para el Congreso Internacional de Traducción Especializada: Entretextos. Valencia, 27-29 de abril de 2016.

HeISS, Christine. (2004) "Dubbing Multilingual Films: A New Challenge?" Meta: Journal des traducteurs 49: 1, pp. 208-220. 
KozlofF, Sarah. (2000) Overhearing film dialogue. Berkeley: University of California Press.

LiPPI-GreEN, Rosina. (2012) English with an Accent: Language, Ideology and Discrimination in the United States. Londres \& Nueva York: Routledge.

LOMEÑa GALIANO, María. (2009) "Variación lingüística y traducción para el doblaje: Mujeres al borde de un ataque de nervios." Entreculturas 1, pp. 275-283. Versión electrónica: <http://www.entreculturas.uma.es/n1pdf/articulo14.pdf>

MARTínEZ-SiERRA, Juan José. (2008) Humor y traducción. Los. Simpson cruzan la frontera. Castellón: Publicaciones Universitat Jaume I.

PiÑEIRO OTERO, M. ${ }^{a}$ Teresa. (2004) "Estereotipos de latinoamericanos en la publicidad radiofónica española." Revista de Comunicación de la SEECI (Sociedad Española de Estudios de la Comunicación Iberoamericana) 11, pp. 41-66. Versión electrónica: <http://www.ucm.es/info/seeci/Numeros/Numero 11/InicioN11. html>

PYM, Anthony. (2000) "Translating Linguistic Variation: Parody and the Creation of Authenticity.” En: Vega, Miguel A. \& Rafael Martín-Gaitero (eds.) 2000. Traducción, metrópoli y diáspora. Madrid: Universidad Complutense de Madrid, pp. 69-75.

RANZATO, Irene. (2010) "Localising Cockney: translating dialect into Italian." En: Díaz Cintas, Jorge; Anna Matamala \& Joselia Neves (eds.) 2010. New Insights into Audiovisual Translation and Media Accessibility. Ámsterdam: Rodopi, pp. 109-122.

REUTNER, Úrsula. (2013) "El dialecto como reto de doblaje: opciones y obstáculos de la traslación de 'Bienvenue chez les Ch'tis'." TRANS: revista de traductología 17, pp. 151-165. Versión electrónica: <http://www.trans.uma.es/trans_17/ Trans17_151-165.pdf>

SHILPA, Davé. (2013) Indian Accents: Brown Voice and Racial Performance in American Television and Film Series: The Asian American Experience. Champaign: University of Illinois Press.

SlobODNík, Duŝan. (1970) "Remarques sur la traduction des dialects." En: Holmes, James S. (ed.) 1970. The Name and Nature of Translation. Bratislava: Slovak Academy of Sciences, pp. 139-143.

STEFFENSEN, Kenn Nakata. (2012) "BBC English with an Accent: 'African' and 'Asian' Accents and the Translation of Culture in British Broadcasting." Meta: journal des traducteurs 57: 2, pp. 510-527.

Teruel Rodríguez, Laura \& Florencio Fernández Cabello-Delgado. (2005)

"Andaluces en la telerrealidad: la audiencia a través del estereotipo del andaluz anónimo en televisión." Comunicar: revista científica iberoamericana de comunicación y educación 25. Versión electrónica: <http://rabida.uhu.es/dspace/ bitstream/handle/10272/6748/Andaluces_en_la_telerrealidad.pdf> 
Whitman-Linsen, Candace. (1992) Through the Dubbing Glass: the Synchronization of American Motion Pictures into German, French, and Spanish. Fráncfort del Meno \& Nueva York: Peter Lang.

\section{Filmografía}

Around the World in Eighty Days (La vuelta al mundo en 80 dias, Michael Anderson 1956)

Beauty and the Beast (Gary Trousdale y Kirk Wise 1991)

Hotel Transylvania (Genndy Tartakovsky 2012)

Puss in Boots (El gato con botas, Chris Miller 2011)

The Big Bang Theory (Chuck Lorre y Bill Prady 2007- )

The Simpsons (Matt Groening 1989- )

Toy Story 3 (Lee Unkrich 2010).

\section{NOTAS BIOGRÁFICAS / BIONOTES}

ANJANA MARTínez TEJERINA es profesora en el Departamento de Traducción de la Universidad Pompeu Fabra desde 2009 y profesora de subtitulación en el posgrado de Traducción Audiovisual de la misma universidad. También colabora como profesora, tutora y evaluadora externa para otras universidades como la Universitat Oberta de Catalunya. Compagina la docencia universitaria con la actividad laboral como traductora autónoma especializada en subtitulación desde hace más de diez años. Entre sus publicaciones destaca el libro El doblaje de los juegos de palabras (2016).

SAMANTHA SÁNCHEZ MARTínEZ es graduada en Traducción e Interpretación con especialidades en inglés y alemán por la Universidad Pompeu Fabra. En esa misma universidad fue seleccionada para colaborar con el Programa de Mentorías, un servicio de formación y acompañamiento dirigido a los estudiantes de los primeros cursos del Grado en Traducción. En la actualidad, tras haber cursado el Máster en Formación de Profesorado de Educación Secundaria, Bachillerato y Formación Profesional de la Universidad Pompeu Fabra y la Universitat Oberta de Catalunya, trabaja como coordinadora y profesora en una reputada academia de inglés.

Anjana Martínez Tejerina has been teaching Translation and Subtitling at Universitat Pompeu Fabra since 2009. She also works as an external tutor and evaluator for other universities such as Universitat Oberta de Catalunya. Besides, she has worked as a freelance translator specialized in subtitling for 
over ten years. In 2016 she published the book El doblaje de los juegos de palabras (The dubbing of wordplay).

Samantha SÁnchez Martínez obtained her Degree in Translation and Interpretation in English and German at Universitat Pompeu Fabra. She was selected by that university to take part in the Programa de Mentorías, a formation and training service in which senior students teach junior students from the same degree. Nowadays, after having studied the Masters on Secondary Education, Language Teaching and Vocational Training at Universitat Pompeu Fabra and Universitat Oberta de Catalunya in order to become an English teacher, she is working as coordinator and teacher in a well-reputed English academy. 
